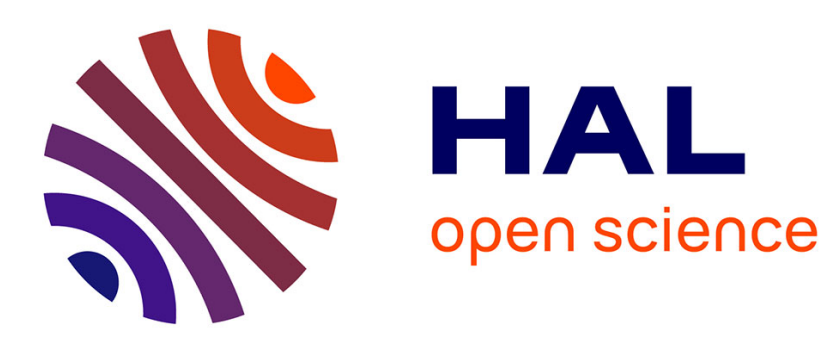

\title{
Power series analysis as a major breakthrough to improve the efficiency of Asymptotic Numerical Method in the vicinity of bifurcations
}

Bruno Cochelin, Médale Marc

\section{- To cite this version:}

Bruno Cochelin, Médale Marc. Power series analysis as a major breakthrough to improve the efficiency of Asymptotic Numerical Method in the vicinity of bifurcations. 2012. hal-00707513

\section{HAL Id: hal-00707513 \\ https://hal.science/hal-00707513}

Preprint submitted on 12 Jun 2012

HAL is a multi-disciplinary open access archive for the deposit and dissemination of scientific research documents, whether they are published or not. The documents may come from teaching and research institutions in France or abroad, or from public or private research centers.
L'archive ouverte pluridisciplinaire HAL, est destinée au dépôt et à la diffusion de documents scientifiques de niveau recherche, publiés ou non, émanant des établissements d'enseignement et de recherche français ou étrangers, des laboratoires publics ou privés. 


\title{
Power series analysis as a major breakthrough to improve the efficiency of Asymptotic Numerical Method in the vicinity of bifurcations
}

\author{
Bruno COCHELIN ${ }^{1,2}$ Marc MEDALE $^{3}$ \\ ${ }^{1}$ LMA, UPR 7051 CNRS \\ 31 chemin Joseph Aiguier \\ 13402 MARSEILLE Cedex 20, France \\ ${ }^{2}$ Centrale Marseille, Technopole de Chateau-Gombert, \\ 13451 MARSEILLE Cedex 20, France \\ ${ }^{3}$ IUSTI, UMR 7343 CNRS-Université Aix-Marseille \\ 5 rue Enrico Fermi, Technopole de Chateau-Gombert, \\ 13453 Marseille Cedex 13, France
}

June 12, 2012

\begin{abstract}
This paper presents the outcome of power series analysis in the framework of the Asymptotic Numerical Method. We theoretically demonstrate and numerically evidence that the emergence of geometric power series in the vicinity of simple bifurcation points is a generic behavior. So we propose to use this hallmark as a bifurcation indicator to locate and compute very efficiently any simple bifurcation point. Finally, a power series that recovers an optimal step length is build in the neighborhood of bifurcation points. The reliability and robustness of this powerful approach is then demonstrated on two application examples from structural mechanics and hydrodynamics.
\end{abstract}

Keywords : Path-following, continuation, bifurcation, power series analysis, Asymptotic Numerical Method.

\section{Introduction}

The main purpose of continuation or path-following algorithms is to map steady state solutions in the parameter space by computing branches of solutions for a given range of control parameters $[18,23]$. In a more detailed 
way, one looks for single or multiple solution ranges, compute bifurcation diagrams and finally perform stability analyses to determine regions of stable and unstable solutions [15, 2]. First order predictor-corrector algorithms (Euler predictor, Newton-Raphson based corrector) with pseudo-arc-length parameterization have been widely used for decades [18, 15, 23]. Nevertheless, step-lengh adaptivity may be in trouble in the vicinity of bifurcation points leading to a weak computational efficiency and sometimes lack of convergence.

An alternate way to first order predictor algorithms stands in high-order predictors that have been introduced in continuation algorithms based on the Asymptotic Numerical Method (ANM). This method consists in a combination of a high-order perturbation method, a discretization technique and a parameterization strategy $[13,3,9,16]$. It is a general and efficient non-linear solution method [12], which has been successfully applied in mainly solid and structural mechanics, but also in few hydrodynamics problems $[8,1,7,17]$. In the ANM the step length is determined a-posteriori from the convergence properties of the series at the current step : it shortens as the non linearities increase and widens as soon as the problem becomes softer, providing a self adaptivity all along the continuation. However, as the continuation approaches to a bifurcation point the self adaptive step length can become very small and the computational efficiency of the ANM procedure becomes significantly penalised. Replacing natural power series by rational ones (Padé approximates) improves the global continuation efficiency as it enables to get step lengths roughly twice as long as in the classical power series representation $[11,5,16]$, but even in that case the step length may be far from its optimal value. The computation of bifurcation points and emanating branches are other particularly important issues. They have been tackled in the ANM framework by computing along the continuation either the zeros of Padé approximates or a scalar bifurcation indicator obtained from an additional perturbed problem solution $[24,28,6,7,17]$. Not only these methods have an extra cost which could be up to twice as the original ANM problem, but also they could be sometimes lacking some robustness.

An alternate promising direction was pioneered in the mid seventies by Van Dyke who initiated the computation of high order perturbation series and their analysis in order to reveal their analytical structure [25]. He proposed several series improvements and completion either to compute them much more efficiently or to extend their range of utility, in an ultimate goal to reveal their underlying mathematical or physical meaning [26, 27]. He made it clear that power series undoubtedly contain numerous relevant information and ways can be found to extract them. But surprisingly, none of Van Dyke's power series analysis has benefited to the ANM yet.

So, in the present work we have analysed the power series that arise at every step of the ANM algorithm and it turns out that in the neighborhood of simple bifurcation points a geometric power series always emerges. Moreover 
this behavior is generic as it does not depend on the problem to be solved. Therefore we propose to use this hallmark as a reliable bifurcation indicator to accurately and cheaply compute any simple bifurcation point. Finally, as soon as it is detected, a new power series that recovers an optimal step length is build in the vicinity of bifurcation points, where the original one is critically penalised in the classical ANM.

The present paper is organized as follows. In Section 2, the theoretical background related to bifurcation analysis is first recalled. Formal derivations of AMN power series are carried out in the framework of perturbed bifurcation analysis. The basic features of ANM power series are then highlighted and discussed. Section 3 is devoted to the way we have implemented geometrical power series extraction from the original power series. The computation of bifurcation points and emanating bifurcation branches are then emphasized. The reliability, robustness and efficiency of this approach is presented in Section 4 throughout two application examples : the buckling of an inextensible elastic beam and the incompressible fluid flow in a symmetric sudden expansion. Finally, conclusions and future work directions are given in Section 5.

\section{Formal derivations of AMN power series in the vicinity of bifurcation points}

In this section we first introduce the minimal needed theoretical background for analysing ANM power series computed near simple bifurcation point. Let $R(u, \lambda)=0$ be an algebraic system of $n$ nonlinear smooth equations where $u \in \mathbb{R}^{n}$ is a vector of state variables and $\lambda \in \mathbb{R}$ a single control or bifurcation parameter. The extended state vector $U=\left[\begin{array}{l}u \\ \lambda\end{array}\right] \in \mathbb{R}^{n+1}$ is introduced for compactness since it includes the parameter $\lambda$ as its last state variable, so the equilibrium system reads :

$$
R(U)=0
$$

without distinguishing between state variables and parameter. Generic solutions of (1) are branches of solutions, ie, one dimensional continua of solution points. In the continuation algorithm based on the ANM, these branches are represented at the current step by the following power series expansion $[13,9,10,12]$ :

$$
U(a)=U_{0}+a U_{1}+a^{2} U_{2}+\cdots+a^{n} U_{n}
$$

with $a$ the path parameter defined in the parameterization equation. In the following, we analyse the case where the starting point $U_{0}$ is a simple bifurcation point, then, a regular point closed to a simple bifurcation. 


\subsection{Branches of solution at a simple bifurcation point}

Let $U_{c}$ be a simple bifurcation point where two branches of solutions cross transversally, ie, with two distinct tangents $U_{t_{1}}$ and $U_{t_{2}}$. The kernel of the jacobian matrix $R_{, U}^{c}$ evaluated at $U_{c}$ is two dimensional,

$$
\mathcal{N}\left(R_{, U}^{c}\right)=\operatorname{Span}\left\{U_{t_{1}}, U_{t_{2}}\right\}, \quad \mathcal{N}\left(R_{, U}^{C T}\right)=\operatorname{Span}\{\psi\} .
$$

while the kernel of the transposed jacobian matrix is one dimensional [15]. The two (normalized) tangent vectors $U_{t_{i}}(i=1,2)$ satisfy the first order approximation of (1) and the so-called "algebraic bifurcation equation" [18], which is the second order approximation of (1) projected on left null-vector $\psi$ of $R_{, U}^{c}$.

$$
\begin{aligned}
& R_{, U}^{c} U_{t_{i}}=0 \\
& \psi^{T} R_{, U U}^{c} U_{t_{i}} U_{t_{i}}=0
\end{aligned}
$$

We also define the (normalized) vector $U_{t_{1}}^{\perp}$ as being inside $\mathcal{N}\left(R_{, U}^{c}\right)$ and orthogonal to $U_{t_{1}}$ (same for $U_{t_{2}}^{\perp}$ ). The branch of solutions emanating from $U_{c}$ along the tangent $U_{t_{1}}$ can be computed in the following way :

$$
U(a)=U_{c}+a U_{t_{1}}+a^{2} U_{2}^{c}+a^{3} U_{3}^{c}+\cdots
$$

where $a=\left(U(a)-U_{c}\right)^{T} U_{t_{1}}$ is the classical pseudo arc-length parameter. Using a Lyapunov-Schmidt decomposition, the vector $U_{p}, p \leq 2$, reads :

$$
U_{p}^{c}=\underbrace{\beta_{p} U_{t_{1}}^{\perp}}_{\in \mathcal{N}\left(R_{, U}^{c}\right)}+\underbrace{V_{p}}_{\in \mathcal{N}^{T}\left(R_{, U}^{c}\right)}
$$

where $V_{P}$ is solution of an augmented invertible linear system (see appendix for detailled expression for the scalar $\beta_{p}$ and the vector $V_{p}$ ). We refer to [3] and [17] for computation of branches of solutions at a bifurcation point in solid or fluid mechanics problems.

\subsection{Perturbed bifurcation analysis at simple bifurcation point : low order approximation}

Small imperfections are always present in experiments, but also in computations owing to finite precision of computers and their numerical solution. Thus, we now pay attention to the following perturbed problem :

$$
R_{p}(U, \mu)=R(U)+\mu P=0
$$

where $P$ is a normalized perturbation vector and $\mu$ a free perturbation amplitude. In the general case $\psi^{T} P \neq 0$, ie, the perturbation vector $P$ is nondegenerate or bifurcation destroying, as opposed to bifurcation preserving 
for the exceptional case where $\psi^{T} P=0$ [22]. Hence, without any loss of generally one can set :

$$
P=\psi+P^{\perp} \text { with } \psi^{T} P^{\perp}=0
$$

With the additionnal free parameter $\mu$ solutions of (7) are no more branches but two dimensional continua of solution points. At the bifurcation point $U_{c}$, the 2D manifold can be found under the form of a two-parameter expansion :

$$
\begin{aligned}
& U=U_{c}+\underbrace{b_{1} U_{t_{1}}+b_{2} U_{t_{2}}}_{\in \mathcal{N}\left(R_{, U}^{c}\right)}+\underbrace{V\left(b_{1}, b_{2}\right)}_{\in \mathcal{N}^{T}\left(R_{, U}^{c}\right)} \\
& \mu=\mu\left(b_{1}, b_{2}\right)
\end{aligned}
$$

where $b_{1}, b_{2}$ are the amplitudes of $U-U_{c}$ on the base vectors $U_{t_{1}}$ and $U_{t_{2}}$ of $\mathcal{N}\left(R_{, U}^{c}\right)$. According to bifurcation theory expansion of $V$ and $\mu$ should begin at order two in $b_{1}, b_{2}$ and the second order of $\mu$ should solve for the "algebraic bifurcation equation" :

$$
\mu\left(b_{1}, b_{2}\right)=b_{1} b_{2} \underbrace{\psi^{T} R_{, U U}^{c} U_{t_{1}} U_{t_{2}}}_{\mu_{1}}
$$

\subsubsection{Perturbed branches}

When the perturbation amplitude is fixed to a given value $\mu_{0}$, generic solutions of (7) get back to one dimensional continua called perturbed branches. They look like the two hyperbolae shown in figure(1) and they are given at low order by the parametric representation :

$$
U\left(b_{1}, b_{2}\right)=U_{c}+b_{1} U_{t_{1}}+b_{2} U_{t_{2}} \quad \text { with } \quad b_{1} b_{2}=\frac{\mu_{0}}{\mu_{1}} .
$$

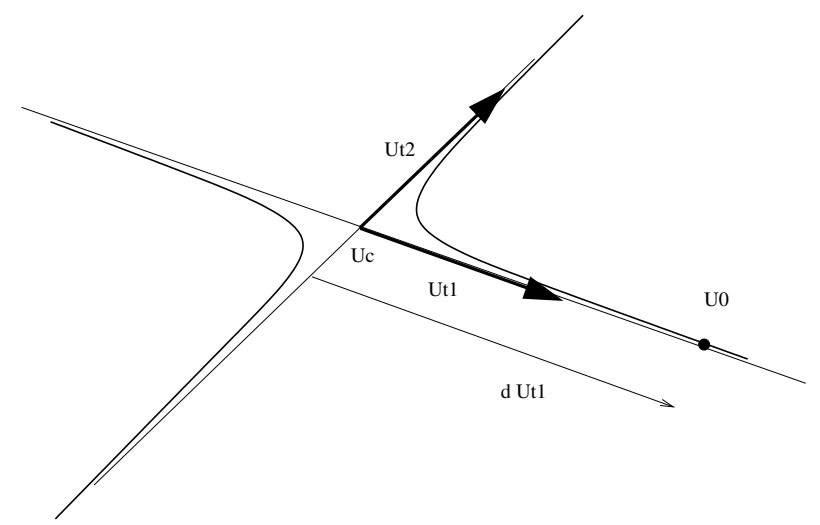

FIgURE 1 - Pertubed bifurcation diagram at lowest order approximation. 


\subsection{Perturbed branch expression from a regular point lo- cated close to a simple bifurcation point}

Let $d$ be a given distance, one sets $b_{1}=d$ and $b_{2}=\frac{\mu_{0}}{\mu_{1} d}$ into (11) to define a regular point :

$$
U_{0}=U_{c}+d U_{t_{1}}+\frac{\mu_{0}}{\mu_{1} d} U_{t_{2}}
$$

at a distance $d$ along $U_{t_{1}}$ from the bifurcation point. We now define the new path parameter $a=d-b_{1}$ and rewrite (11) as :

$$
U(a)=U_{0}+a\left(-U_{t_{1}}\right)+\frac{\mu_{0}}{\mu_{1} d} \frac{\frac{a}{d}}{\left(1-\frac{a}{d}\right)} U_{t_{2}}
$$

This last equation gives a parametric expression of the perturbated branch from the regular solution point $U_{0}(a=0)$ toward the bifurcation point $U_{c}$ $(a>0)$. The rational fraction that appears in $U_{t_{2}}$ 's amplitude has a pole for $a=d$, which makes the perturbed branch turn in the direction of $U_{t_{2}}$ near the bifurcation point. Notice that when $\mu_{0}$ is very small the amplitude for $U_{t_{2}}$ is also very small except when $a$ is very close to $d$.

This simple reasoning that leads to (13) already shows the key point of the present analysis : for any non zero imperfection the (vector) function $U(a)$ has a singularity for $a=d$, ie, for a positive real value of the parameter a and the nature of the singularity is of the kind $\left(1-\frac{a}{d}\right)^{-1}$. The Taylor series of such a rational fraction is $: \frac{a}{d}+\left(\frac{a}{d}\right)^{2}+\left(\frac{a}{d}\right)^{3}+\cdots$. It represents a geometrical progression whose common ratio is $\frac{1}{d}$ and radius of convergence $d$. These results are globally in line with the properties of the ANM series when the expansion point is close to a bifurcation point. However, the picture is not complete because (13) is only a first order approximation.

\subsection{Extention to higher order approximation}

To extend the previous results at high orders we begin with the derivation of a two parameter expansion of the $2 \mathrm{D}$ manifold under the form :

$$
\begin{aligned}
& U\left(a_{1}, a_{2}\right)=U_{c}+a_{1} U_{t_{1}}+a_{2} U_{t_{1}}^{\perp}+a_{1}^{2} V_{20}+a_{1} a_{2} V_{11}+a_{2}^{2} V_{02} \\
& +a_{1}^{3} V_{30}+a_{1}^{2} a_{2} V_{21}+a_{1} a_{2}^{2} V_{12}+a_{2}^{3} V_{03} \\
& +\cdots \\
& \mu\left(a_{1}, a_{2}\right)=a_{1}^{2} \mu_{20}+a_{1} a_{2} \mu_{11}+a_{2}^{2} \mu_{02} \\
& +a_{1}^{3} \mu_{30}+a_{1}^{2} a_{2} \mu_{21}+a_{1} a_{2}^{2} \mu_{12}+a_{2}^{3} \mu_{03} \\
& +\cdots
\end{aligned}
$$

where $a_{1}$ and $a_{2}$ are defined as the amplitude of $U-U_{c}$ on the base vector $U_{t_{1}}$ and $U_{t_{1}}^{\perp}$ of $\mathcal{N}\left(R_{, U}^{c}\right)$. In the perspective to analyse ANM power series, the choice of $\left(a_{1}, a_{2}\right)$ parameters is better than $\left(b_{1}, b_{2}\right)$, which were the best one for a first order analysis. All the vectors $V_{i j}$ 's are orthogonal to $U_{t_{1}}$ 
and $U_{t_{1}}^{\perp}$, their expression have been reported in appendix for brevity in the presentation.

The next step is to rewrite the $2 \mathrm{D}$ manifold by taking $a_{1}$ and $\mu$ as parameters instead of $a_{1}$ and $a_{2}$. For this we follow the procedure proposed by Damil and Potier-Ferry in [13] to get :

$$
\begin{aligned}
U\left(a_{1}, \mu\right)= & U_{c}+a_{1} U_{t_{1}}+a_{1}^{2} U_{2}^{c}+a_{1}^{3} U_{3}^{c}+\cdots \\
& +\frac{\mu}{\mu_{11}}\left(\frac{1}{a_{1}} U_{t_{1}}^{\perp}+U_{0,1}+a_{1} U_{1,1}+a_{1}^{2} U_{2,1}+\cdots\right) \\
& +\frac{\mu^{2}}{\mu_{11}^{2}}\left(\frac{1}{a_{1}^{3}} U_{t_{1}}^{\perp}+\frac{1}{a_{1}^{2}} U_{-2,2}++\frac{1}{a_{1}} U_{-1,2}+\cdots\right) \\
& +\frac{\mu^{2}}{\mu_{11}^{3}}\left(\frac{1}{a_{1}^{5}} U_{t_{1}}^{\perp}+\cdots\right)
\end{aligned}
$$

The first line of (16) exactly corresponds to (5), the case with no imperfection. Notice also that singular terms of the kind $\frac{1}{a_{1}^{p}}$ appear in subsequent lines of this expression.

\subsubsection{First order truncation in $\mu$}

In our ANM continuation process imperfections appear because the solution points where the series are computed do not exactly satisfy (1) but only up to a required user-defined precision $\varepsilon_{R}$, ie, $U_{0}$ is a solution point if $\left\|R\left(U_{0}\right)\right\|<\varepsilon_{R}$. The ANM series evaluated at $U_{0}$ are indeed the exact formal solution of the perturbed problem $R(U)-R\left(U_{0}\right)=0$. In applications, $\varepsilon_{R}$ is generally set to a very small value, hence, the imperfection amplitude $\mu_{0}$ considered herafter will also be very small. As a consequence, we begin this analysis in limiting to a first order truncature in $\mu$.

\subsubsection{Perturbed branch expression form a regular point $U_{0}$}

As above we set the imperfection amplitude $\mu$ to a fixed value $\mu_{0}$ to define perturbed branches. Then, we deport the expansion point from $U_{c}$ to $U_{0}$ by taking $a=d-a_{1}$ to get :

$$
\begin{aligned}
U(a)= & U_{0}+a U_{t_{1}}^{0}+a^{2} U_{2}^{0}+a^{3} U_{3}^{0}+\cdots \\
& +\frac{\mu 0}{\mu_{11}}\left(\frac{a}{d(d-a)} U_{t_{1}}^{\perp}+a U_{1,1}^{0}+a^{2} U_{2,1}^{0}+\cdots\right)
\end{aligned}
$$

The new vector $U_{i}^{0}$ and $U_{i, j}^{0}$ (at $U_{0}$ ) can be easily deduced from the $U_{i}^{c}$ and $U_{i, j}\left(\right.$ at $\left.U_{c}\right)$ of $(16)$ and $d$.

Let $r$ be the radius of convergence of the first line of (17), ie, the series for the perfect branch. Let us scale the path parameter $a$ by $r$, so that the radius of convergence of the perfect branch becomes unity and the norm of the series coefficient become of order of unity. Denoting $\bar{a}=\frac{a}{r}$, we get the 
final expression for the perturbed branches :

$$
\begin{aligned}
U(\bar{a})= & U_{0}+\bar{a}\left(r U_{t_{1}}^{0}\right)+\bar{a}^{2}\left(r^{2} U_{2}^{0}\right)+\bar{a}^{3}\left(r^{3} U_{3}^{0}\right)+\cdots \\
& +\frac{\mu 0}{\mu_{11}}\left(\frac{\bar{a} \frac{r}{\bar{d}}}{d\left(1-\bar{a} \frac{r}{d}\right)} U_{t_{1}}^{\perp}+\bar{a}\left(r U_{1,1}^{0}\right)+\bar{a}^{2}\left(r^{2} U_{2,1}^{0}\right)+\cdots\right)
\end{aligned}
$$

The ANM method does not give access to (18) but only to its powers series. Expanding the rational fraction of (18) one can get the following expression for the series coefficient $\bar{U}_{p}^{0}$ at order $p$ of the rescaled ANM expansion evaluated near a simple bifurcation point :

$$
\bar{U}_{p}^{0}=r^{p} U_{p}^{0}+\frac{\mu_{0}}{\mu_{11}} r^{p} U_{p, 1}^{0}+\frac{\mu_{0}}{\mu_{11} d}\left(\frac{r}{d}\right)^{p} U_{t_{1}}^{\perp}
$$

The first term $r^{p} U_{p}^{0}$ corresponds to the perfect case situation $\mu_{0}=0$. Because of the rescalling, its norm is of order of unity. The second term $\frac{\mu_{0}}{\mu_{11}} r^{p} U_{p, 1}^{0}$ is a the first order correction of the previous one due to the imperfection and its norm is also of order of unity. The third term $\frac{\mu_{0}}{\mu_{11} d}\left(\frac{r}{d}\right)^{p} U_{t_{1}}^{\perp}$ is associated with the singularity brought by the perturbed bifurcation. Its amplitude is a product of the small imperfection $\mu_{0}$ with the $p$ power of $\frac{r}{d}$, which can be very large when $d<<r$.

It follows the important results : when the distance $d$ between $U_{0}$ and $U_{c}$ is smaller than $r$, ie, the bifurcation point is inside the radius of convergence of the "perfect" series, the last term of (19) dominates the series at high order whatever the imperfection magnitude $\mu_{0}$. Moreover, at high order the series behaves like a geometrical progression with a common ratio $\frac{r}{d}$ (or $\frac{1}{d}$ for the non scaled series), and the series term $\bar{U}_{p}^{0}$ (or $U_{p}^{0}$ for the nonscaled series) becomes colinear to the vector $U_{t_{1}}^{\perp}$.

In other words, when $d<r$ and $\mu_{0} \neq 0$, the pole of the rational fraction in (18) (or (17)) becomes the nearest singularity of $U(\bar{a})$ and set the radius of convergence to $d$, instead of $r$ for the perfect case $\mu_{0}=0$. This is perfectely in line with the observation made on ANM series at expansion point close to a simple bifurcation point, as it will be shown on examples below.

\subsubsection{Return to high order terms in $\mu$}

Let us now come back to the high order $\mu$ term in (16) that have been suppressed above, claiming that $\mu$ is very small in our applications. One question still remains : what is the influence of more singular terms such as $\frac{\mu_{0}^{2}}{\mu_{11}^{2}} \frac{1}{a_{1}^{3}}$ as compared to the less singular term $\frac{\mu_{0}}{\mu_{11}} \frac{1}{a_{1}}$ retained in the previous analysis?. To answer it, let us analyse the Taylor series of the following toy model :

$$
f(x)=\underbrace{\frac{1}{1-x}}_{f_{1}(x)}+\underbrace{10^{-3} \frac{1}{(1-x)^{3}}}_{f_{2}(x)} .
$$


According to Van Dyke [25] the radius of convergence, sign pattern in the Taylor series coefficients and values of coefficients at high order are both governed by the nearest singularity of the function. When two singularities are at the same distance, as in this example, the most singular ones will impose itself (here $f_{2}$ ), rather than the one with the largest amplitude (here $f_{1}$ ). However, the prominence of $f_{2}$ only occurs at very high order as it is reported in table 1 , because of its small amplitude $10^{-3}$.

\begin{tabular}{|c|c|c|c|c|c|c|c|c|c|}
\hline order & 1 & 2 & 3 & $\ldots$ & 43 & 44 & $\ldots$ & 99 & 100 \\
\hline$f_{1}$ & 1 & 1 & 1 & $\ldots$ & 1 & 1 & $\ldots$ & 1 & 1 \\
\hline$f_{2}$ & 0.003 & 0.006 & 0.01 & $\ldots$ & 0.99 & 1.035 & $\ldots$ & 5.050 & 5.151 \\
\hline
\end{tabular}

TABLE 1 - Taylor coefficients for the function $f_{1}=\frac{1}{1-x}$ and $f_{2}=10^{-3} \frac{1}{(1-x)^{3}}$.

In (16), the most singular terms have a much smaller amplitude than the less singular ones when $\mu_{0}$ is very small. Practically, with $\mu_{0}=10^{-8}$ and a truncature around order 20 , these more singular terms do not show up in our analysis.

\section{Implementation of power series analysis in the Asymptotic Numerical Method}

It has been previously pointed out that power series analysis is twofold in the framework of the Asymptotic Numerical Method : (i) it provides an accurate and computationally efficient way to detect and compute bifurcation points (solution at bifurcation points); (ii) it enables us to construct an enhanced power series that recovers an optimal step length in the vicinity of bifurcation points where the classical ANM is otherwise drastically penalised. So the way we have implemented these key points are now presented.

\subsection{Detection of geometric progression in the ANM}

Any geometric progression can be characterized by two quantities : its common ratio and its scale factor, which is here a field vector. So, the proposed ANM algorithm includes at every continuation step an analysis of the last vector sequence to check if they make up a geometric progression or not. Let $U_{n-3}, U_{n-2}, U_{n-1}, U_{n}$ be the last four field vector sequence in the ANM power series (2) computed up to an order of troncature $n$. Then, the detection of a geometric progression checks for collinearity and proportionality of 
this vector sequence. This test reads as follows :

$$
\begin{aligned}
& \text { for } n-3 \leq p \leq n-1: \alpha_{p}=\left(U_{p} \cdot U_{n}\right) /\left(U_{n} \cdot U_{n}\right) \text { and } U_{p}^{\perp}=U_{p}-\alpha_{p} U_{n} \\
& \text { if } \sum_{p=n-3}^{n-2}\left(\left(\alpha_{p}^{1 /(n-p)}-\alpha_{n-1}\right) / \alpha_{n-1}\right)^{2}<\varepsilon_{g p_{1}} \text { and } \sum_{p=n-3}^{n-1}\left\|U_{p}^{\perp}\right\| /\left\|U_{p}\right\|<\varepsilon_{g p_{2}} \\
& \text { then a geometric progression is detected, so one computes : } \\
& \text { - its common ratio : } \frac{1}{\alpha}=\frac{1}{\alpha_{n-1}} \\
& \text { - its scale factor : } U_{n} \alpha^{n}
\end{aligned}
$$

According to section 2, $\alpha$ is the distance to the closest bifurcation point and the normalised scale factor $\left(\frac{U_{n}}{\left\|U_{n}\right\|}\right)$ is the vector $U_{t_{1}}^{\perp}$ previously defined.

In our calculations setting the two numerical threshold values to $\varepsilon_{g p_{1}} \approx$ $10^{-3}$ and $\varepsilon_{g p_{2}} \approx 10^{-6}$ gave us reliable and robust bifurcation detection test.

\subsection{Enhanced power series to recover optimal step length}

Let us now suppose a geometric progression has been just detected at the highest orders of the perturbation method with the proposed test (21). Then, the original power series (2) can be splitted in two parts : a geometric power series and the remaining part from the orignal one, as follows :

$$
\begin{array}{r}
U(a)=U_{0}+a \hat{U}_{1}+a^{2} \hat{U}_{2}+\cdots+a^{n-1} \hat{U}_{n-1}+ \\
{\left[\frac{a}{\alpha}+\left(\frac{a}{\alpha}\right)^{2}+\cdots+\left(\frac{a}{\alpha}\right)^{n-1}+\left(\frac{a}{\alpha}\right)^{n}\right] U_{n} \alpha^{n}}
\end{array}
$$

where $\hat{U}_{p}=U_{p}-\alpha^{n-p} U_{n}$ for $0<p \leq n-1$. Introducing the formal calculation of the geometrical power series together with a completion at infinity, and using $\frac{U_{n}}{\left\|U_{n}\right\|}=U_{t_{1}}^{\perp}$, one finally gets :

$$
U(a)=U_{0}+a \hat{U}_{1}+a^{2} \hat{U}_{2}+\cdots+a^{n-1} \hat{U}_{n-1}+\frac{\frac{a}{\alpha}}{1-\frac{a}{\alpha}}\left\|U_{n}\right\| \alpha^{n} U_{t_{1}}^{\perp}
$$

The power series analysis introduced in section 2 enables us to understand the underlying issues related to these two parts. The first part of (23) is the power series that actually describes the perfect branch, whereas the second part, i.e., the geometric power series accounts for the interaction and amplification of any imperfection with the other branch that crosses the present one at the next bifurcation point. Then, the key point to recover an optimal step length over this continuation step is to only consider the former power series to accurately represent the current branch. So the enhanced power series representation reads :

$$
U(a)=U_{0}+a \hat{U}_{1}+a^{2} \hat{U}_{2}+\cdots+a^{n-1} \hat{U}_{n-1}
$$

which results from an additive singularity extraction of (2) in the same way as formerly proposed in [25]. 


\subsection{Computation of the solution vector at bifurcation points}

Once equipped with the enhanced power series (24), one can accurately locate the nearest singularity, i.e., simple bifurcation point and compute both the solution vector and its two tangent vectors at the bifurcation point. Recalling that it corresponds to the radius of convergence of the power series, which in the present case is the inverse of the common ratio of the geometric progression, the solution vector at bifurcation point is therefore computed by inserting $a=\alpha$ in (24) :

$$
U_{c}=U(a=\alpha)=U_{0}+\alpha \hat{U}_{1}+\alpha^{2} \hat{U}_{2}+\cdots+\alpha^{n-1} \hat{U}_{n-1}
$$

Furthermore, the tangent vector to the current branch is computed at the bifurcation point as follows :

$$
U_{t_{1}}=\frac{\partial U}{\partial a}(a=\alpha)=\hat{U}_{1}+2 \alpha \hat{U}_{2}+\cdots+(n-1) \alpha^{n-2} \hat{U}_{n-1}
$$

Finally, the tangent vector to the other branch $U_{t_{2}}$ is easily and cheaply computed as a linear combination of the known vectors $U_{t_{1}}$ and $U_{t_{1}}^{\perp}$, meanwhile satisfying the algebraic bifurcation equation (4-b).

$$
\begin{aligned}
& U_{t_{2}}=\beta U_{t_{1}}+\gamma U_{t_{1}}^{\perp} \\
& \psi^{T} R_{, U U}^{c} U_{t_{2}} U_{t_{2}}=0
\end{aligned}
$$

As far as the bifurcation point and the two tangent vectors associated to the crossing branches are completely determined, branch switching can be performed at wish in the course of continuation.

\section{Application examples}

For illustrating the performance of the proposed algorithm, we first consider the buckling problem of an inextensible elastic beam. It is a small size discrete problem coming from solid mechanics, which presents an elaborate bifurcation diagram with many interconnected branches of solutions. On the other hand the proposed algorithm has also been implemented in a high performance framework [21] to deal with large size discrete problems such those that arise in many fluid mechanics or coupled fluid flow and heat transfer problems. We have performed several validation tests and present below the algorithm accuracy and efficiency for an incompressible fluid flow problem in a symmetric sudden expansion.

\subsection{Buckling analysis of an elastica beam}

We consider the axial compression of an inextensible elastic beam, often referred to as the Euler beam or Elastica, for which we adopt clampedclamped boundary conditions. Let $s \in[0,1]$ be the nondimensional curvilinear coordinate, $(x(s), y(s))$ the position of the beam centerline, $\theta(s)$ the 
rotation of the cross-section and $m(s)$ the bending moment (all nondimentional). The equilibrium positions of the beam are given by the following ODE system :

$$
\begin{aligned}
& x^{\prime}=\cos (\theta) \\
& y^{\prime}=\sin (\theta) \\
& \theta^{\prime}=m \\
& m^{\prime}=-4 \pi^{2}(P \sin (\theta)+T \cos (\theta))
\end{aligned}
$$

with the boundary conditions $x(0)=0, y(0)=0, \theta(0)=0, m(0)=2 \pi C$ (at left) and $y(1)=0, \theta(1)=0$ (at right). The axial applied force $P$ is here the control parameter, $T$ and $C$ are the lateral and torque reactions at the boundary. This ODE system is transformed into a discrete problem (276 d.o.f) by applying an orthogonal collocation method with cubic polynomial interpolation [14]. The bifurcation diagram is made of a trivial compressive solution ( $x=s, y=0, \theta=0, m=0)$ from which bifurcated branches emanate at bifurcation points $P=\{1 ; 2.04 ; 4 ; \ldots\}$. Additional branches of solutions interconnect these bifurcated branches at "secondary" bifurcation points.

\subsubsection{Detailled analysis of continuation and bifurcation detection}

Starting from a known point $U_{s 0}$ on the first bifurcated branch, we perform 4 steps of ANM continuation with a troncature order of 20 and a user-required precision $\varepsilon_{R}=10^{-7}$. Two geometrical series are detected by the test (21) and the associated bifurcation points are marked with circles on the bifurcation diagram reported in figure 2. The bifurcation (I) detected at the first continuation step corresponds to the intersection of the first bukling branch with the trivial compressive solution. The imperfection amplitude $\mu_{0}$ is here very small $\left(\mu_{0}=2.142310^{-15}\right)$ because the start point $U_{s 0}$ is very accurate $\left(R\left(U_{s 0}\right)=1.461510^{-12}\right.$ and $\left.\mu_{11}=-0.0698\right)$. Even though, since the ratio $\frac{r}{d}$ is large (about 17 in that case) the geometrical series end up imposing itself at orders higher than 13 . The end point of the first step $U_{s 1}$ is computed by considering (24) to recover an optimal step length. The original series (2) would have deliver a much shorter step length as it is reported in figure 2. We note in passing that this exemple is similar to the one in [20] where the author says that a judicious re-ordering of the series terms leads to suprising improvements.

For the second continuation step, ie, with the series issued from $U_{s 1}$, no geometrical serie was detected by the algorithm, even though the bifurcation point (II) is located inside the radius of convergence. The reason in the present case is that the ratio $\frac{r}{d}$ is not large enough so that the term $\left(\frac{r}{d}\right)^{p}$ compensates a so tiny $\mu_{0}$ value. In other words the singularity brought by the bifurcation (II) is actually the nearest one but as its amplitude is small and the other singularities are not so far the geometrical series does not succeed to impose itself. Hence, a standard ANM step is performed from 

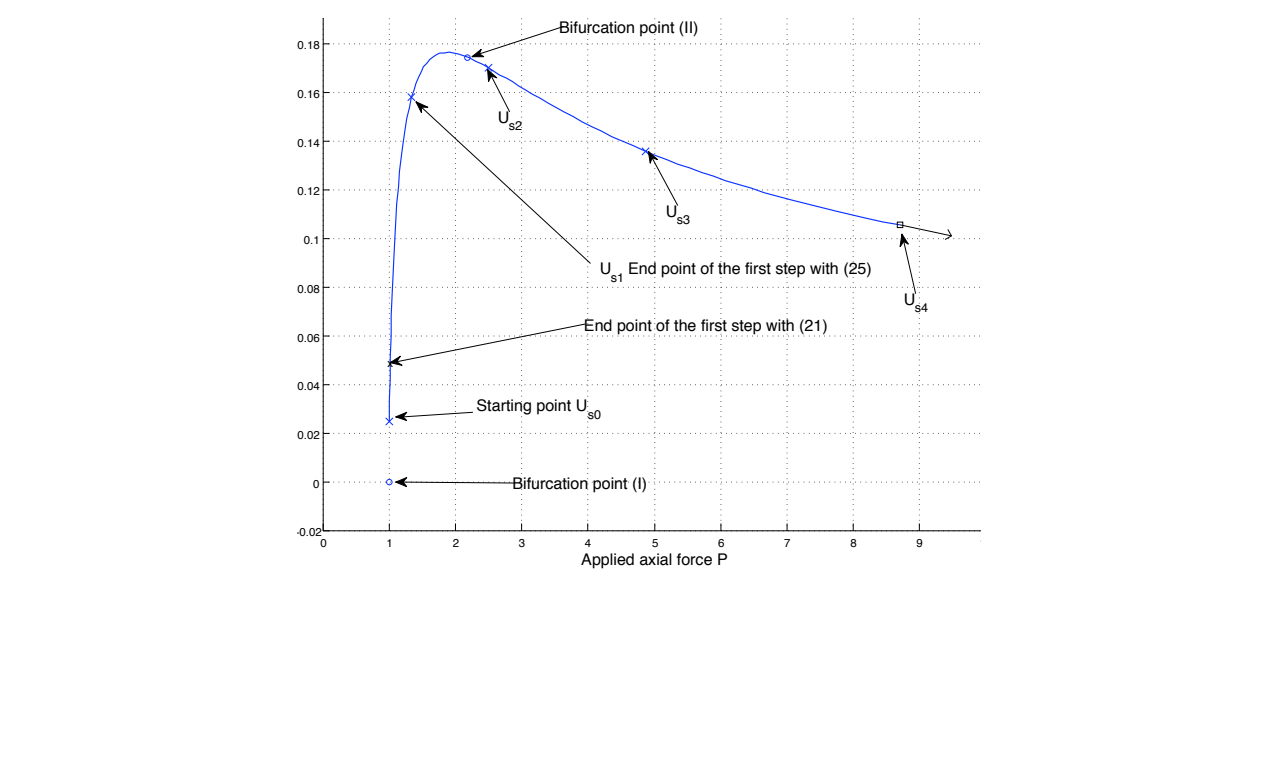

FiguRE 2 - Buckling analysis of an elastica beam. Bifurcation diagram after 4 continuation steps (top); deformed beam shapes at points $U_{s i}(0 \leq i \leq 4$, bottom).

$U_{s 1}$ to $U_{s 2}$. This could be somehow disappointing because the bifurcation is not detected, but as $U_{s 2}$ is close to the bifurcation the detection will happen at the next step. Indeed, at the third step of continuation (with the series computed from $\left.U_{s 2}\right)$ one gets : $R\left(U_{s 2}\right)=1.67810^{-7}, \mu_{0}=9.517410^{-14}$, $\mu_{11}=-3.827710^{-4}, \frac{r}{d}=15.873$ is large enough so a geometrical series correponding to (II) is easily detected.

In summary the scenario described in this example bring to the fore two important algorithm features : (i) the closer the bifurcation point from the starting one, the easier it is detected; (ii) end of step points computed by standard ANM continuation algorithm are generally close to bifurcation points [4].

\subsubsection{Performance of the proposed algorithm on intricate bifur- cation diagrams}

The bifurcation diagrams presented in figures (2) and (3) have been obtained with the MANLAB interactive package [19], which supports the proposed ANM continuation algorithm for bifurcation detection and branch switching. Along the 65 ANM continuation steps the proposed criterion (21) has detected 12 geometrical series, from which one can get accurate bifurcation locations and bifurcating tangents with no additional cost. Only 65 jacobian matrix factorizations have been performed to get the bifurcation 
diagram plotted in figure (3), a performance that could not be achieved neither with classical predictor-corrector continuation algorithms nor previous ANM continuation ones.
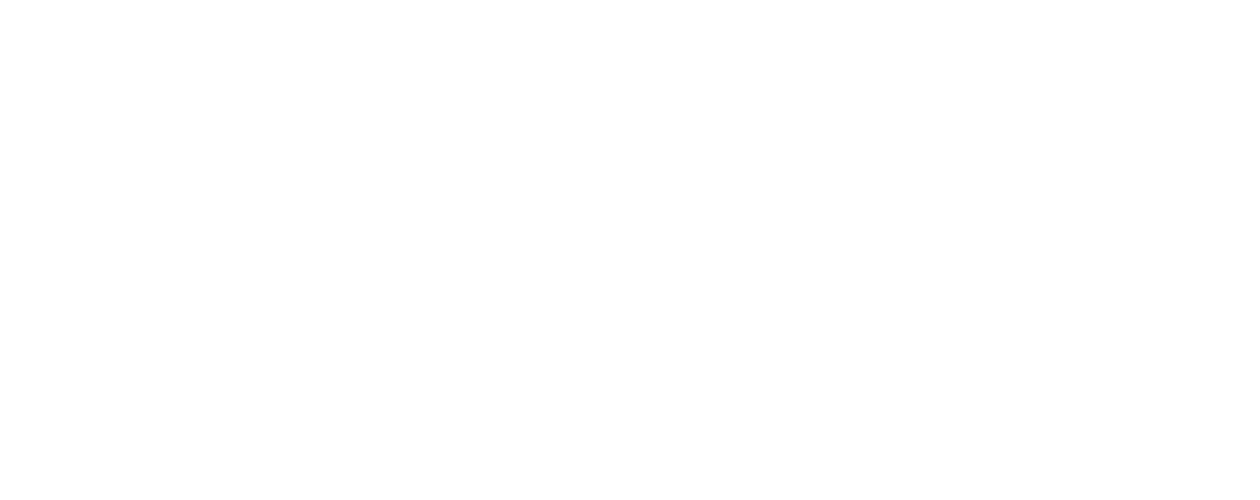

FiguRE 3 - Bifurcation diagram for the first two buckling branches (left). The close loop that connects these two branches at four bifurcation points has been presented separately for clarity of the figure (right).

\subsection{Two-dimensional incompressible fluid flow in a symmet- ric sudden expansion}

We consider the computation of steady state incompressible fluid flows in a two-dimensional channel with a symmetric sudden expansion, see figure 4 . The computational domain has an entrance channel length of $2 h(A B=$ $G H)$, a symmetric expansion aspect ratio of $2(D E=2 A H)$ and a developing channel that extends on $50 h(C D=E F)$. The flow enters the computational domain with a Poiseuille velocity profile $\left(u(-2 h, z)=U\left(1-4 z^{2} / h^{2}\right)\right.$ and $w(-2 h, z)=0 ;-h / 2 \leq z \leq h / 2)$. The boundary condition at the outlet $(D E)$ is set to homogeneous Neumann condition (stress free), and no slip boundary condition is imposed at all solid walls. This flow configuration has

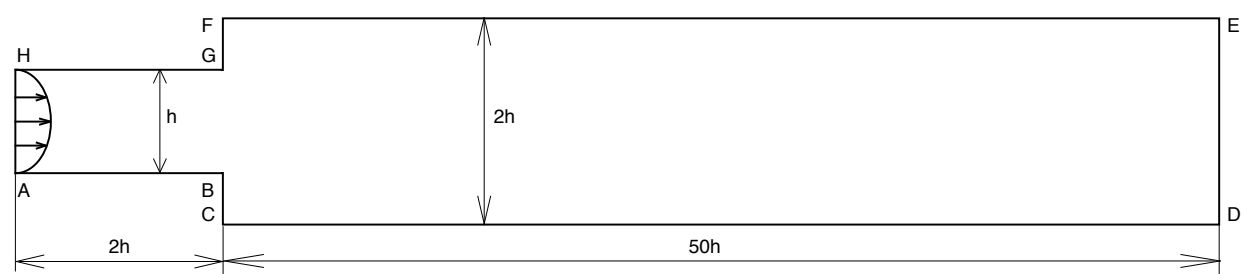

FiguRE 4 - Sketch of the two-dimensional symmetric sudden expansion channel flow.

been undertaken in the literature with both experimental and numerical approaches and a large amount of critical Reynolds number at which symmetry 
breaking occurs have been published. Several of them have been gathered in [17] for comparison purpose, so the Reynolds number is here again defined as $R e=U h / \nu$ ( $\nu$ is the fluid kinematic viscosity).

The present continuation algorithm has been run on this problem with four successively refined meshes, uniformly spaced in both directions, as indicated in table 2. Indeed, the standard Galerkin finite element formulation used to discretize the incompressible Navier-Stokes equation [21] can achieve accurate fluid flow computations provided the local Reynolds number does not exceed a critical value $\left(R e_{l} \leq 2 \operatorname{Coe} f_{p}, R e_{l}=\sqrt{(u \Delta x)^{2}+(w \Delta z)^{2}} / \nu\right.$ and $\operatorname{Coef}_{p}$ is a coefficient which depends on the approximation degree and the problem under consideration). Therefore, the finer the mesh, the higher the computational accuracy up to a stability Reynolds number limit defined as $R e_{\max }=2 \operatorname{Coef}_{p} h / \sqrt{(\Delta x)^{2}+(\Delta z)^{2}}$. Table 2 presents for each mesh the number of degree of freedom $\left(N_{d o f}\right)$, the corresponding spatial resolution $(\Delta x, \Delta z)$ and finally the theoretical Reynolds number limit $\left(R e_{\max }\right)$ beyond which spurious oscillation could appear without any stabilization $\left(\operatorname{Coef}_{p}=16\right)$. The continuation has been performed from $R e=0$ up to $R e_{\max }$ for the four meshes considered, taking an order of troncature of the power series up to 30 and numerical parameters $\delta=10^{-9}, \varepsilon_{g p 1}=10^{-3}$ and $\varepsilon_{g p 2}=10^{-6}$. Furthermore, a Newton-Raphson correction has been performed subsequently to any ANM predictor step whose residual $L_{2}$ norm exceeded $\varepsilon_{R}=10^{-6}$.

\begin{tabular}{|c|c|c|c|c|}
\hline Mesh \# & M1 & M2 & M3 & M4 \\
\hline$N_{\text {dof }}$ & 106242 & 421378 & 1678338 & 6699010 \\
\hline$\Delta x, \Delta z$ & $h / 8, h / 16$ & $h / 16, h / 32$ & $h / 32, h / 64$ & $h / 64, h / 128$ \\
\hline$R e_{\max }$ & 458 & 916 & 1832 & 3664 \\
\hline
\end{tabular}

TABLE 2 - Meshes considered in the computations of the $2 \mathrm{D}$ fluid flow in a symmetric sudden expansion.

Meanwhile the symmetry breaking occurs throughout a pitchfork bifurcation, if one keeps going on the fundamental symmetric branch beyond the first bifurcation, one can encounter subsequent steady state bifurcations. We have reported their values in table 3 for each mesh, together with the number of continuation step at which they have been computed. Finally, we have also reported in table 3 the bifurcation values obtained in [17], whenever there were obtained on a much coarser mesh $\left(N_{d o f}=5542\right)$ than those considered in the present work. A very satisfactory agreement is obtained for the first and second bifurcation values, but a larger difference appears for the third one. The reason of this discrepancy at large Reynolds numbers is likely due to the coarse mesh used in [17], which should have been operated beyond its accuracy limit at these relatively large flow magnitudes.

Up to our best knowledge, only the first three bifurcations have ever 


\begin{tabular}{|c|c|c|c|c|c|c|}
\hline Bif\# & 1 & 2 & 3 & 4 & 5 & 6 \\
\hline M1 & $215.01(6)$ & $538.49(9)$ & - & - & - & - \\
\hline M2 & $215.47(6)$ & $537.57(9)$ & $966.06(11)$ & - & - & - \\
\hline M3 & $215.73(6)$ & $536.81(9)$ & $967.19(11)$ & $1447.16(13)$ & $1988.71(16)$ & - \\
\hline M4 & $216.67(6)$ & $536.06(9)$ & $967.96(11)$ & $1442.13(13)$ & $1983.92(16)$ & $2420.33(26)$ \\
\hline \hline$[17]$ & 215.63 & 537.40 & 946.47 & - & - & - \\
\hline
\end{tabular}

TABLE 3 - Computed bifurcation points for the 2D fluid flow in a symmetric sudden expansion for the four meshes considered.

been reported in the literature [17]. However we have computed six steady state bifurcations on the fundamental branch for a computational domain that extends on $50 h$ downstream the sudden expansion. In order to characterize them, the streamlines and the right null eigenvector related to each bifurcation point have been plotted in figures 5-8. One can observe that as the Reynolds number increases, the two symmetric recirculation zones stretch further and further in the streamwise direction and the locus of the maximun w-component eigenvector is accordingly repealed more and more downstream in the channel. Therefore, based on the present computations it clearly appears that the number of subsequent bifurcations is closely related to the channel length. Moreover, at the sixth bifurcation point the most unstable mode associated to the right null eigenvector spreads beyond the computation domain outlet (located at $L=50 h$ ). Therefore, the presently computed Reynolds number value at this bifurcation point could not be taken for granted unless a longer computational domain has been considered.

\section{Conclusion}

This paper presents both formal analysis and numerical evidence that a geometric power series proportional to the residual error always emerges in ANM power series close to simple bifurcation points. The discover of this remarquable feature enabled us to introduce in the ANM framework an entirely new and original algorithm to detect and compute simple bifurcation points. Indeed, as soon as a geometrical series is detected in the classical ANM power series, it can be advantageously substracted from it to accurately locate and compute the neighboring simple bifurcation point and its associated tangent vectors with no extra cost. Moreover, the resulting enhanced power series recovers an optimal step length, which represents a significant computational efficiency improvement with respect to the classical ANM algorithm that is otherwise severely penalised in the vicinity of bifurcation points. Branch switching has now become entirely reliable and efficient so the overall ANM continuation algorithm has definitely become highly competitive in matrix factorization counts as it has been experienced throughout a wide range of 


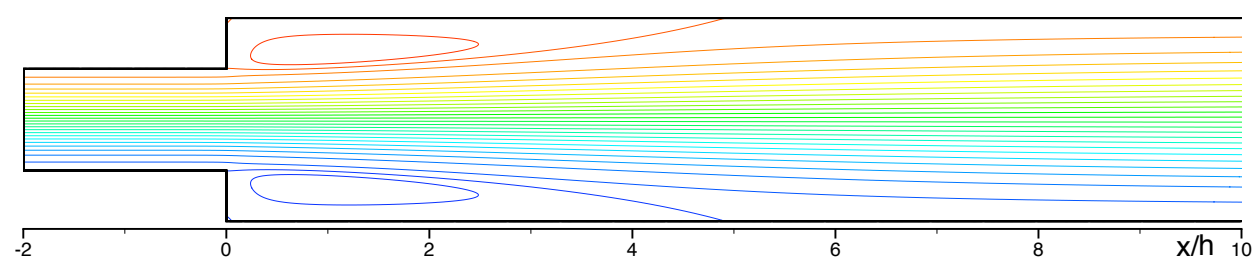

(a) Streamlines.

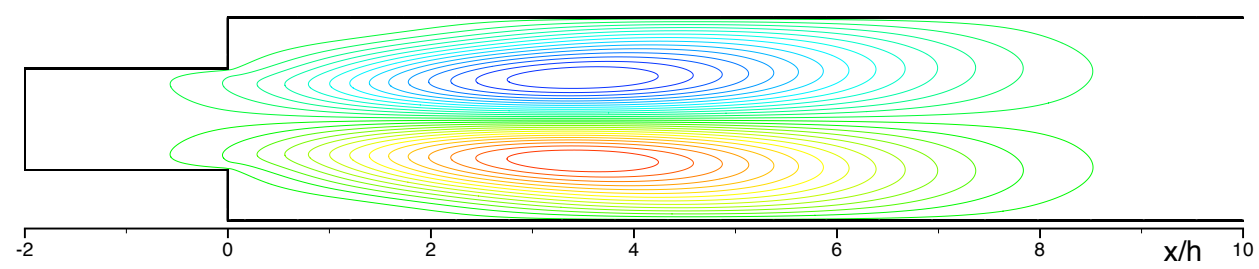

(b) u-component of the right null eigenvector.

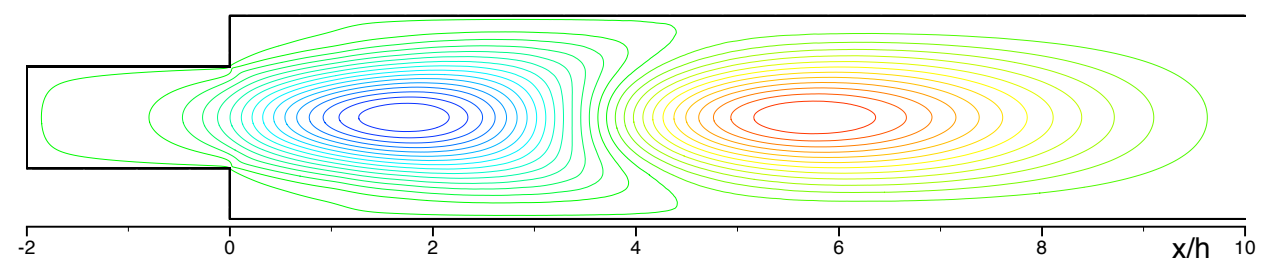

(c) v-component of the right null eigenvector.

FigURE 5 - Streamlines and right null eigenvector at first bifurcation point.

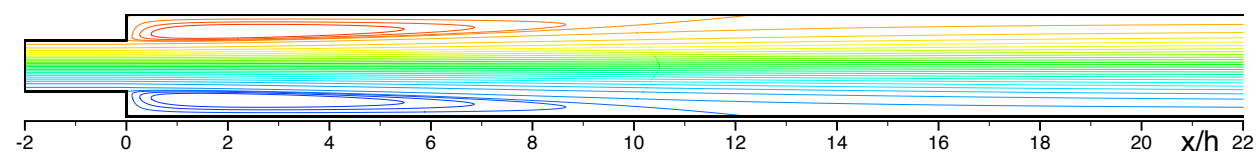

(a) Streamlines.

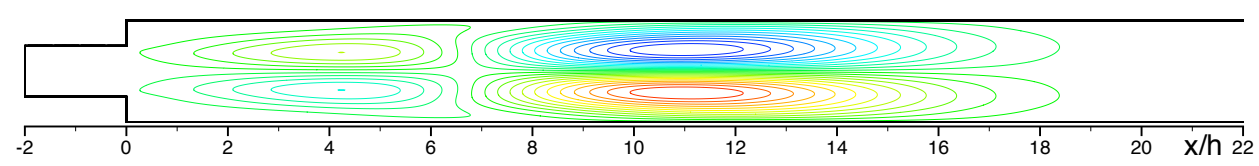

(b) u-component of the right null eigenvector.

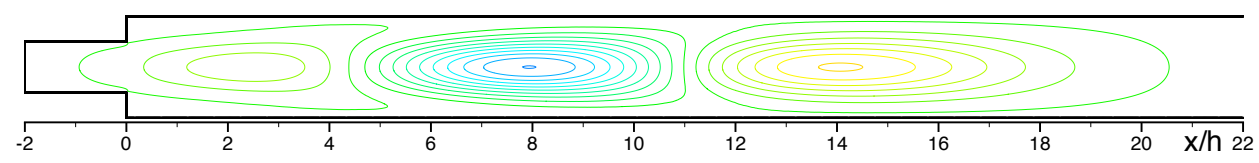

(c) v-component of the right null eigenvector.

FigURE 6 - Streamlines and right null eigenvector at second bifurcation point. 


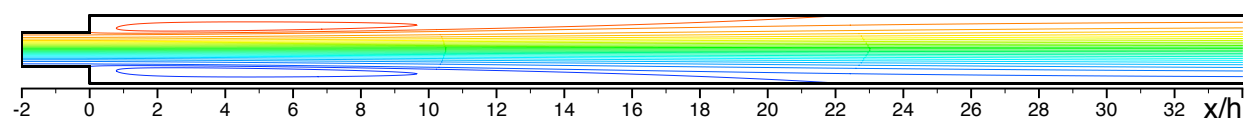

(a) Streamlines.

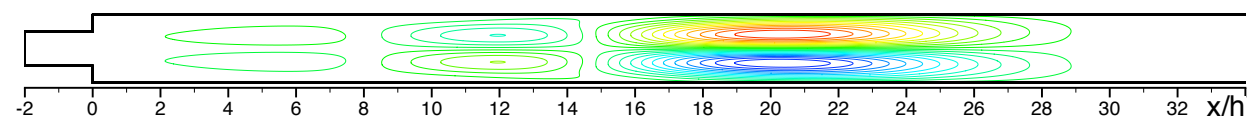

(b) u-component of the right null eigenvector.

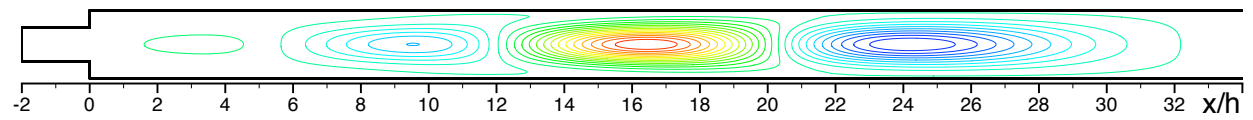

(c) v-component of the right null eigenvector.

FiguRE 7 - Streamlines and right null eigenvector at third bifurcation point.

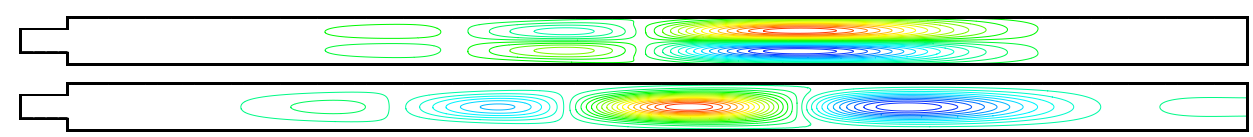

(a) u-v components of the right null eigenvector at forth bifuraction point.

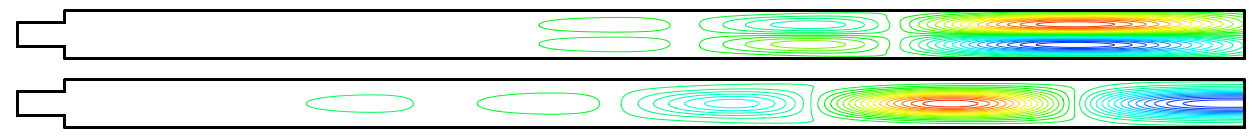

(b) $\mathrm{u}-\mathrm{v}$ components of the right null eigenvector at fifth bifuraction point.

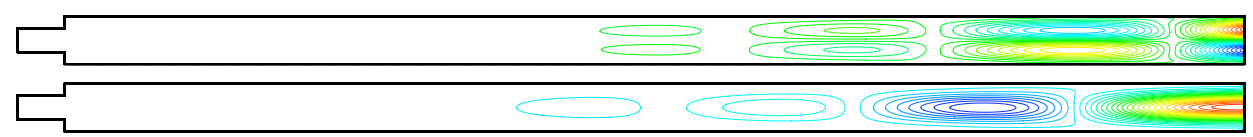

(c) u-v components of the right null eigenvector at sixth bifuraction point.

Figure 8 - Right null eigenvector at fourth (a), fifth (b) and sixth (c) bifurcation points. 
application examples.

In conclusion this work provides an additional evidence that high order perturbation series contain valuable information that it can be worth to extracted to reveal their underlying mathematical or physical meaning, as it was claimed long time ago by Van Dyke [25]. Whatever we were already able to use the radius of convergence or at least the range of utility of the power series to get an optimal continuation step length [12], we are now able to detect the nearby presence of a simple bifurcation. Undoubtedly many relevant information could be extracted from ANM series and we believe it is worth to keep a sustaining research effort in that direction. The analysis of bifurcation points with higher multiplicity is the next step to be considered as a natural prolongation of the present work.

\section{Appendix}

More details are given in this appendix on the formal derivations introduced in section 2 that lead to the final important expression (16). For simplicity, one assumes that the vector equation (1) is polynomial and quadratic in $U$, so it reads :

$$
R(U)=L 0+L(U)+Q(U, U)=0 .
$$

where $L 0$ is a constant vector, $L($.$) a linear operator and Q(., *)$ a bilinear operator. This assumption is not mandatory but very convenient since it enables an easy derivation of the Taylor series which are at the heart of the analysis. Most scientific problems are not polynomial and quadratic at first, but they can be written under the form (29) provided that additional state variables and equations are added, see for instance [12].

Branches of solution at a simple bifurcation point : introducing (5) into (29) and collecting terms with the same power of $a$, one gets :

$$
\begin{aligned}
& R_{, U}^{c} U_{1}=0 \\
& R_{, U}^{c} U_{2}+Q\left(U_{1}, U_{1}\right)=0 \\
& R_{, U}^{c} U_{3}+Q\left(U_{1}, U_{2}\right)+Q\left(U_{2}, U_{1}\right)=0 \\
& \vdots \\
& R_{, U}^{c} U_{p}+\sum_{r=1}^{p-1} Q\left(U_{r}, U_{p-r}\right)=0
\end{aligned}
$$

with the notation $U_{1}=U_{t_{1}}$. According to the definition of $a$, we have $U_{p}^{T} \cdot U_{t_{1}}=0$ for $p \geq 2$. The following Lyapunov-Schmidt decomposition is done for each unknown vector $U_{p}$ with $p \geq 2$,

$$
U_{p}=\underbrace{\alpha_{p} U_{t_{1}}+\beta_{p} U_{t_{1}}^{\perp}}_{\in \mathcal{N}\left(R_{, U}^{c}\right)}+\underbrace{V_{p}}_{\in \mathcal{N}^{T}\left(R_{, U}^{c}\right)=\mathcal{R}\left(R_{, U}^{C T}\right)}
$$


where $U_{t_{1}}^{\perp} \in \mathcal{N}\left(R_{, U}^{c}\right)$ and is orthogonal to $U_{t_{1}}$. Finally, $\alpha_{p}=0, V_{p}$ is the unique solution of $\left[\begin{array}{cc}R_{, U}^{c} & \psi \\ U_{t_{1}}^{T} & 0 \\ U_{t_{1}}^{\perp T} & 0\end{array}\right]\left[\begin{array}{l}V_{p} \\ \mu_{p}\end{array}\right]=\left[\begin{array}{c}-\sum_{r=1}^{p-1} Q\left(U_{r}, U_{p-r}\right) \\ 0 \\ 0\end{array}\right]$ and

$$
\begin{aligned}
& \beta_{2}=-\frac{\psi^{T}\left\{Q\left(U_{t_{1}}, V_{2}\right)+Q\left(V_{2}, U_{t_{1}}\right)\right\}}{\psi^{T}\left\{Q\left(U_{t_{1}}, U_{t_{1}}^{\perp}\right)+Q\left(U_{t_{1}}^{\perp}, U_{t_{1}}\right)\right\}} \\
& \beta_{3}=-\frac{\psi^{T}\left\{Q\left(U_{t_{1}}, V_{3}\right)+Q\left(V_{2}, V_{2}\right)+Q\left(V_{3}, U_{t_{1}}\right)\right\}}{\psi^{T}\left\{Q\left(U_{t_{1}}, U_{t_{1}}^{\perp}\right)+Q\left(U_{t_{1}}^{\perp}, U_{t_{1}}\right)\right\}} \\
& \vdots \\
& \beta_{p}=-\frac{\psi^{T}\left\{Q\left(U_{t_{1}}, V_{p}\right)+\sum_{r=2}^{p-1} Q\left(U_{r}, U_{p+1-r}\right)+Q\left(V_{p}, U_{t_{1}}\right)\right\}}{\psi^{T}\left\{Q\left(U_{t_{1}}, U_{t_{1}}^{\perp}\right)+Q\left(U_{t_{1}}^{\perp}, U_{t_{1}}\right\}\right.}
\end{aligned}
$$

Perturbed bifurcation analysis at a simple bifurcation point : inserting (14) and (15) into (7) and collecting terms with the same power in $a_{1}$ and $a_{2}$, one gets :

$$
\begin{array}{llll}
\text { order } a_{1}^{2} & R_{, U}^{c} V_{20} & +\mu_{20} P & +Q\left(U_{t_{1}}, U_{t_{1}}\right)=0 \\
\text { order } a_{1} a_{2} & R_{, U}^{c} V_{11} & +\mu_{11} P & +Q\left(U_{t_{1}}, U_{t_{1}}^{\perp}\right)+Q\left(U_{t_{1}}^{\perp}, U_{t_{1}}\right)=0 \\
\text { order } a_{2}^{2} & R_{, U}^{c} V_{02} & +\mu_{02} P & +Q\left(U_{t_{1}}^{\perp}, U_{t_{1}}^{\perp}\right)=0 \\
& & & \\
\text { order } a_{1}^{3} & R_{, U}^{c} V_{30} & +\mu_{30} P & +Q\left(U_{t_{1}}, V_{20}\right)+Q\left(V_{20}, U_{t_{1}}\right)=0 \\
\text { order } a_{1}^{2} a_{2} & R_{, U}^{c} V_{21} & +\mu_{21} P & +Q\left(U_{t_{1}}, V_{11}\right)+Q\left(V_{11}, U_{t_{1}}\right) \\
& & & +Q\left(U_{t_{1}}^{\perp}, V_{20}\right)+Q\left(V_{20}, U_{t_{1}}^{\perp}\right)=0 \\
& & & \\
\vdots & & & \\
\text { order } a_{1}^{i} a_{2}^{j} & R_{, U}^{c} V_{i j} & +\mu_{i j} P & +F_{i j}^{n l}=0
\end{array}
$$

$V_{i j}$ and $\mu_{i j}$ are the unique solution of the (non singular) linear system

$$
\left[\begin{array}{cc}
R_{U}^{c} & P \\
U_{t_{1}}^{T} & 0 \\
U_{t_{1}}^{\perp T} & 0
\end{array}\right]\left[\begin{array}{c}
V_{i j} \\
\mu_{i j}
\end{array}\right]=\left[\begin{array}{c}
-F_{i j}^{n l} \\
0 \\
0
\end{array}\right]
$$

with

$$
\begin{aligned}
& \mu_{20}=0 \\
& \mu_{11}=-\psi^{T}\left\{Q\left(U_{t_{1}}, U_{t_{1}}^{\perp}\right)+Q\left(U_{t_{1}}^{\perp}, U_{t_{1}}\right)\right\} \\
& \mu_{02}=-\psi^{T}\left\{Q\left(U_{t_{1}}^{\perp}, U_{t_{1}}^{\perp}\right)\right) \\
& \mu_{30}=-\psi^{T}\left\{Q\left(U_{t_{1}}, V_{20}\right)+Q\left(V_{20}, U_{t_{1}}\right)\right\}
\end{aligned}
$$


The $V_{p}, \beta_{p}$ of (31) are connected to the $V_{i j}, \mu_{i j}$ of (14) and (15) by

$$
\begin{array}{ll}
V_{2}=V_{20} & \beta_{2}=-\frac{\mu_{30}}{\mu_{11}} \\
V_{3}=V_{30}+\beta_{2} V_{11} & \beta_{3}=-\frac{\mu_{40}+\beta_{2} \mu_{21}+\beta_{2}^{2} \mu_{02}}{\mu_{11}} \\
V_{4}=V_{40}+\beta_{2} V_{21}+\beta_{3} V_{11}+\beta_{2}^{2} V_{02} & \beta_{4}=-\frac{\mu_{50}+\beta_{3} \mu_{21}+\beta_{2} \mu_{31}+2 \beta_{2} \beta_{3} \mu_{02}+\beta_{2}^{2} \mu_{12}}{\mu_{11}}
\end{array}
$$

Parametrisation of the 2D manifold with $a_{1}$ and $\mu$. Hereafer, (15) is reverted to express $a_{2}$ as function of $a_{1}$ and $\mu$. We follow the procedure proposed by Damil and Potier-Ferry in [13], ie, we rescale $a_{1}, a_{2}$ and $\mu$ as follows :

$$
a_{1}=\eta A_{1} \quad a_{2}=\eta^{2} A_{2} \quad \mu=\eta^{3}
$$

and assume the following expansion of $A_{2}$ in $A_{1}$ and $\eta$ :

$$
A_{2}\left(A_{1}, \eta\right)=f_{0}\left(A_{1}\right)+\eta f_{1}\left(A_{1}\right)+\eta^{2} f_{2}\left(A_{1}\right)+\eta^{3} f_{3}\left(A_{1}\right)+\ldots
$$

where $f_{0}, f_{1}, f_{2}, \ldots$ are function of $A_{1}$ only, to be determined. Inserting (38) into (15), collecting term with the same power of $\eta$ and solving the equations yields :

$$
\begin{aligned}
f_{0}\left(A_{1}\right) & =\beta_{2} A_{1}^{2}+\frac{1}{\mu_{11} A_{1}} \\
f_{1}\left(A_{1}\right) & =\beta_{3} A_{1}^{3}+c_{0,1} \frac{1}{\mu_{11}}+c_{-3,2} \frac{1}{\mu_{11}^{2} A_{1}^{3}} \\
f_{2}\left(A_{1}\right) & =\beta_{4} A_{1}^{4}+c_{1,1} \frac{A_{1}}{\mu_{11}}+c_{-2,2} \frac{1}{\mu_{11}^{2} A_{1}^{2}}+c_{-5,3} \frac{1}{\mu_{11}^{3} A_{1}^{5}}:
\end{aligned}
$$

where the $c_{i, j}$ are

$$
\begin{aligned}
& c_{0,1}=-\frac{\mu_{21}+2 \beta_{2} \mu_{02}}{\mu_{11}} \\
& c_{-3,2}=-\frac{\mu_{02}}{\mu_{11}} \\
& c_{1,1}=-\frac{\mu_{31}+c_{0,1} \mu_{21}+\left(\beta_{2}+c_{0,1} \beta_{3}\right) \mu_{02}+2 \beta_{2} \mu_{12}}{\mu_{11}}
\end{aligned}
$$

Coming back to the original parameters $a_{1}, a_{2}, \mu$, expression for $a_{2}$ is :

$$
\begin{aligned}
a_{2}\left(a_{1}, \mu\right)= & \beta_{2} a_{1}^{2}+\beta_{3} a_{1}^{3}+\beta_{4} a_{1}^{4}+\ldots \\
& +\frac{\mu}{\mu_{11}}\left(\frac{1}{a_{1}}+c_{0,1}+c_{1,1} a_{1}+\ldots\right) \\
& +\frac{\mu^{2}}{\mu_{11}^{2}}\left(c_{-3,2} \frac{1}{a_{1}^{3}}+c_{-2,2} \frac{1}{a_{1}^{2}}+\ldots\right) \\
& +\frac{\mu^{3}}{\mu_{11}^{3}}\left(c_{-5,3} \frac{1}{a_{1}^{5}} \ldots\right)
\end{aligned}
$$

Reporting (41) into (14) and accounting for (36) and (31), one finally ends up with the expression (16). 
Acknowledgement : The authors are thankful to Dr D. Garajeu for very fruitful discussions. The second author acknowledges CNRS for providing computing resources on its IBM-SP6 supercomputer at IDRIS and its SGIAltix one at CINES under project 026455.

\section{Références}

[1] C. Allery, J.M. Cadou, A. Hamdouni, and D. Razafindralandy. Application of the Asymptotic Numerical Method to the Coanda effect study. Revue Eur. Eléments Finis, 13 :57-77, 2004.

[2] E. L. Allgower and K. G. Georg. Numerical Continuation Methods. An introduction. Springer-Verlag, New York, 1990.

[3] L. Azrar, B. Cochelin, N. Damil, and M. Potier-Ferry. An Asymptotic Numerical Method to compute the post-buckling behaviour of elastic plates and shells. Int. J. Num. Meth. Eng., 36 :1251-1277, 1993.

[4] S. Baguet and B. Cochelin. On the behaviour of the ANM continuation in the presence of bifurcation. Communication in Numerical Methods in Engineering, 19 :459-471, 2003.

[5] A. Baker and P. Graves-Morris. Padé approximants. Cambridge University Press, 1996.

[6] E.H. Boutyour, H. Zahrouni, M. Potier-Ferry, and M. Boudi. Asymptotic Numerical Method for buckling analysis of shell structures with large rotation. Journal of Computational and Applied Mathematics, $168: 77-85,2004$.

[7] J.M. Cadou, B. Cochelin, and M. Potier-Ferry. A numerical method to compute bifurcation points in fluid mechanics. Eur. J. Mechanics B Fluids, 25 :234-254, 2006.

[8] J.M. Cadou, M. Potier-Ferry, B. Cochelin, and N. Damil. Asymptotic Numerical Method for stationary Navier-Stokes equations and with Petrov-Galerkin formulation. Int. J. Num. Meth. Eng., 50 :825-845, 2001.

[9] B. Cochelin. A path following technique via an Asymptotic Numerical Method. Computers and Structures, 53 :1181-1192, 1994.

[10] B. Cochelin, N. Damil, and M. Potier-Ferry. The Asymptotic Numerical Method : an efficient perturbation technique for non-linear structural mechanics. Revue Eur. Eléments Finis, 5 :415-442, 1994.

[11] B. Cochelin, N. Damil, and M. Potier-Ferry. Asymptotic Numerical Method and Padé approximants for non-linear elastic structures. Int. J. Num. Meth. Eng., 37 :1187-1213, 1994.

[12] B. Cochelin, N. Damil, and M. Potier-Ferry. Méthode Asymptotique Numérique (in french). Hermès - Lavoisier, 2007. 
[13] N. Damil and M. Potier-Ferry. A new method to compute perturbed bifurcations : application to the buckling of imperfect elastic structures. Int. J. Eng. Sci., 28 :943-957, 1990.

[14] E. Doedel. Lecture notes on numerical analysis of nonlinear equations. In B. Krauskopf, H.M. Osinga, and J. Galan-Vioque, editors, Numerical Continuation Methods for dynamical systems, pages 1-49. Springer, 2007.

[15] E. Doedel, H. B. Keller, and J. P. Kernevez. Numerical analysis and control of bifurcation problems (i) bifurcation in finite dimensions. Int. J. Bifurcation and Chaos, 3 :493-520, 1991.

[16] A. Elhage-Hussein, M. Potier-Ferry, and N. Damil. A numerical continuation method based on Padé approximants. Int. J. Solids and Structures, 37 :6981-7001, 2000.

[17] Y. Guevel, H. Boutyour, and J.-M. Cadou. Automatic detection and branch switching methods for steady bifurcation in fluid mechanics. Journal of Computational Physics, 230 :3614-3629, 2011.

[18] H. B. Keller. Numerical solution of bifurcation and nonlinear eigenvalue problems. Academic Press, New York, 1977.

[19] Manlab. An interactive path-following and bifurcation analysis software, http ://manlab.lma.cnrs-mrs.fr/, 2012.

[20] P. O. Mattei. High-order perturbation expansion for the spectral analysis of fluid-loaded vibrating structure. Acta Acustica united with Acustica, 93 :303-313, 2007.

[21] M. Medale and B. Cochelin. A parallel computer implementation of the Asymptotic Numerical Method to study thermal convection instabilities. Journal of Computational Physics, 228 :8249-8262, 2009.

[22] M. Potier-Ferry. Foundations of elastic post-buckling theory in Buckling and Post-buckling, Lecture notes in Physics, volume 288. SpringerVerlag, Berlin, 1987.

[23] R. Seydel. Nonlinear Computation. J. Franklin Inst., 334B :1015-1047, 1997.

[24] A. Tri, B. Cochelin, and M. Potier-Ferry. Résolution des équations de Navier-Stokes et détection des bifurcations stationnaires par une Méthode Asymptotique Numérique. European Journal of Finite Elements, 5(4) :415-442, 1996.

[25] M. Van Dyke. Analysis and improvement of perturbation series. J. Mech. Appl. Math., 27(423-450), 1974.

[26] M. Van Dyke. Computer extension of perturbation series in fluid mechanics. SIAM J. Appl. Math., $28: 720-734,1975$.

[27] M. Van Dyke. Perturbation methods in fluid mechanics. The Parabolic Press, Stanford, California, 1975. 
[28] P. Vannucci, B. Cochelin, N. Damil, and M. Potier-Ferry. An Asymptotic Numerical Method to compute bifurcating branches. International Journal for Numerical Methods in Engineering, 41 :1365-1389, 1998. 